\title{
Dieta de la lagartija de los gramadales Microlophus thoracicus icae en el valle del río lca, Perú
}

\author{
Diet of the grass lizard Microlophus thoracicus icae in the Ica river valley, Peru
}

\section{José Pérez Z., ${ }^{1,2}$ Emilio Fuentes ${ }^{2}$ y Juan Jordán²}

\author{
1 Laboratorio de Estudios en Biodiversidad, Departamento de Ciencias Biológicas y Fisiológicas, Facultad de Ciencias y Filosofía, Universidad Peruana \\ Cayetano Heredia, Perú. \\ 2 Departamento de Herpetología, Museo de Historia Natural. Universidad Nacional de San Marcos, Perú. \\ Email José Pérez: jose.perez.z@upch.pe \\ Email Emilio Fuentes: emiliofuentesgarcia@hotmail.com \\ Email Juan Jordán: juan.jordan@gmail.com
}

\begin{abstract}
Resumen
Se evaluó la dieta de la lagartija de los gramadales Microlophus thoracicus icae en tres localidades del Valle del río Ica, Perú. La dieta de este saurio se caracterizó por un importante consumo de material vegetal, principalmente foliolos del árbol Prosopis spp., e invertebrados, especialmente insectos como hormigas y larvas de insectos. No se registraron relaciones significativas entre el tamaño corporal de los individuos y el número total de presas consumidos, ni el volumen total consumido. Los juveniles, hembras y machos de $M$. $t$. icae no presentaron diferencias significativas en el número de hormigas o larvas de insectos consumidos. Tampoco se registraron diferencias significativas en la proporción de material vegetal consumido por juveniles, hembras y machos. Sin embargo, el volumen de material vegetal consumido fue mayor para machos y hembras, comparado con juveniles. No se observan diferencias evidentes entre las dietas de juveniles, hembras y machos en el análisis multivariado. La amplitud del nicho trófico para M. $t$. icae fue de $\mathrm{B}_{i j}=6.97$. El consumo de material vegetal e invertebrados es importante para juveniles y adultos de $M$. $t$. icae, por lo tanto, no se observa una marcada variación etaria en la dieta de los individuos evaluados. Esta especie de saurio presentaría una gran plasticidad en su dieta (omnívora) influenciada principalmente por la variación local de la oferta de los recursos alimenticios. Las posibles consecuencias del consumo de una dieta tan variada podrían incluir características particulares en sus endoparásitos, estrategias y eficiencia de forrajeo, termorregulación, morfología, entre otros.
\end{abstract}

Palabras Clave: Dieta; Saurio; Desierto Costero Peruano; omnivoría; Lagartija de gramadales.

\section{Abstract}

The diet of grass lizard, Microlophus thoracicus icae, was evaluated in three localities of the Ica River Valley, Peru. The dietary pattern was characterized by high consumption of vegetable material, mainly Prosopis spp. leaves, and invertebrates as ants and insect larvae. No significant relationships were found between body size, number of prey eaten or volume consumed. The juvenile, male and female M. t. icae not showed significant differences regarding number of ants or insect larvae consumed, neither on the proportion consumed of plant material. However, total volume of plant material was different between males and females, compared to juveniles. Multivariate analysis showed no evident difference in the diets of juveniles, males and females. Trophic niche amplitude for $M$. $t$. icae was $\mathrm{B}_{i j}=6.97$. The consumption of plant material and invertebrates is important for both juvenile and adult iguanas, therefore; no clear age difference in diet was observed in the individuals studied. This species would present great diet plasticity (omnivory) influenced by the local variation of food resources. Possible consequences of a varied diet may include particular characteristics of its parasites, foraging strategies and efficiency, thermoregulation, morphology, among others.

Keywords: Diet; Saurian; Desierto Costero Peruano; omnivory; Tschudi's Pacific Iguana.

Citación:

Pérez Z. P., E. Fuentes \& J. Jordán. 2015. Dieta de la lagartija de los gramadales Microlophus thoracicus icae en el valle del río Ica, Perú. Revista peruana de biología 22(2): 233 - 238 (Octubre 2015). doi: http:// dx.doi.org/10.15381/rpb.v22i2.11357

$\begin{array}{ll}\text { Presentado: } & 26 / 01 / 2015 \\ \text { Aceptado: } & 25 / 04 / 2015\end{array}$

Publicado online: $14 / 10 / 2015$
Información sobre los autores: JPZ, EF: realizaron el diseño experimental; EF: realizó el trabajo de campo; JPZ, EF, JJ: analizaron los datos; redactaron, revisaron y aprobaron el manuscrito. Los autores no incurren en conflictos de intereses.

Los autores no incurren en conflictos de intereses.

Permisos de colecta: Instituto Nacional de Recursos Naturales (INRENA) N¹30-2006 INRENA-IFFS-DCB.

Journal home page: http://revistasinvestigacion.unmsm.edu.pe/index.php/rpb/index

(c) Los autores. Este artículo es publicado por la Revista Peruana de Biología de la Facultad de Ciencias Biológicas, Universidad Nacional Mayor de San Marcos. Este es un artículo de acceso abierto, distribuido bajo los términos de la Licencia Creative Commons Atribución-NoComercial-Compartirlgual 4.0 Internacional.(http://creativecommons.org/licenses/by-nc-sa/4.0/), que permite el uso no comercial, distribución y reproducción en cualquier medio, siempre que la obra original sea debidamente citadas. Para uso comercial, por favor póngase en contacto con editor.revperubiol@gmail.com. 


\section{Introducción}

La mayoría de las especies de saurios presentes en el Desierto Costero peruano presentan una dieta principalmente insectívora (Dixon \& Wright 1975, Pefaur \& López-Tejeda 1983, Pérez \& Jahncke 1998, Pérez 2005, Pérez \& Balta 2007, Pérez \& Balta 2011, Pérez et al. 2012), sin embargo, también ha sido reportado el consumo de material vegetal en especies como los gecónidos Phyllodactylus angustidigitus y P. gerrhopygus (Pérez \& Balta 2011), tropiduridos Microlophus peruvianus (Pérez \& Balta 2007), M. thoracicus (Dixon \& Wright 1975; Pérez \& Balta 2007) y $M$. tigris (Pérez 2005), y teidos como Dicrodon guttulatum (Pollack et al. 2007, Van Leeuwen et al. 2011).

Entre estas especies destaca la lagartija de los gramadales, Microlophus thoracicus Tschudi 1845, ya que es indicada como una especie que presenta un elevado consumo de material vegetal específicamente en la etapa adulta (Dixon \& Wright 1975). Debido a esta característica estos autores diferencian a M. thoracicus de la mayoría de especies del género Microlophus, sin embargo no existen estudios que contribuyan con mayor información sobre la dieta de esta especie y la posible variación ontogenética en el consumo de material vegetal mencionado por Dixon y Wright (1975).

Microlophus (anteriormente Tropidurus [Frost, 1992]) thoracicus es conocida con el nombre común de "lagartija de los gramadales" (Icochea 1998), y se reconocen tres subespecies (Dixon \& Wright 1975, Carrillo e Icochea 1995), donde M. thoracicus icae presenta una distribución sureńa entre los departamentos de Arequipa e Ica (Dixon \& Wright 1975), incluyendo el valle de Ica. Los objetivos de esta investigación fueron: i) caracterizar cuantitativamente la dieta de $M$. thoracicus icae, ii) cuantificar el consumo de material vegetal por parte de $M$. thoracicus icae, iii) identificar la potencial variación ontogenética del consumo del material vegetal entre juveniles y adultos.

\section{Área de estudio}

Las colectas fueron realizadas durante enero del 2002 en tres localidades en el valle del río Ica: San José de los Molinos, Huacachina y Ocucaje. El valle del río Ica se caracteriza por la presencia de especies herbáceas que constituyen un $80 \%$ de las especies registradas, seguidas por especies leñosas, semileñosas y cactoides (Roque \& Cano 1999). El árbol "huarango" Prosopis pallida fue una de las especies características de las localidades evaluadas, y las poblaciones de $M$. thoracicus icae estudiadas estuvieron asociadas a esta especie de árbol.

La herpetofauna diurna registrada en las áreas evaluadas estuvo conformada por el cañán Dicrodon heterolepis de la Familia Teiidae, lagartija de los gramadales Microlophus thoracicus icae, la lagartija de los arenales Microlophus theresiae y la lagartija de las Lomas Microlophus tigris de la Familia Tropiduridae.

\section{Materiales y métodos}

Los individuos de $M$. thoracicus icae fueron capturados durante el período de actividad de la especie (horario diurno), específicamente en horarios con alta probabilidad de capturar individuos que ya se hubieran alimentado, y que sus contenidos estomacales no estuvieran muy digeridos. Este período corresponde aproximadamente a tres o cuatro horas después de observarse el cielo despejado.
Para cada lagartija capturada se registró la longitud corporal hocico-cloaca (SVL) con una regla Vernier $(0.1 \mathrm{~mm})$. Cada individuo colectado fue sacrificado mediante la inoculación de alcohol $70 \%$ al corazón. Todos los estómagos fueron extraídos, y almacenados en alcohol $70 \%$ para preservar adecuadamente el contenido estomacal. Todos los especímenes fueron preservados siguiendo los procedimientos estándares en herpetología (Pisan \& Villa 1974).

Se realizó el análisis cada contenido estomacal con la ayuda de un microscopio estereoscópico, obteniéndose datos del número, volumen y frecuencia de cada ítem alimentario consumido. Los ítems alimentarios fueron identificados hasta nivel de orden para los insectos, y hasta clases para otros invertebrados. Para realizar un análisis adecuado se definieron categorías específicas como Hymenóptera (alados), Hymenóptera (Hormigas), Larvas de insectos, Partes de Artrópodos No Identificados (PANI) y material vegetal. En la categoría Material Vegetal se agrupan todas las partes consumidas de las plantas.

Se analizaron los datos de dieta considerando tres categorías: juveniles, hembras y machos, identificando a los juveniles como menores de $50 \mathrm{~mm}$ de longitud corporal (SVL), y diferenciando los sexos de los adultos por las coloraciones típicas de los machos, que presentan dorso amarillento y gula roja (Dixon \& Wright 1975).

Se estimó el volumen de cada presa consumida mediante la fórmula (Dunham 1983):

$$
\mathrm{V}=4 / 3 \pi(\operatorname{largo} / 2)(\text { ancho/2 })^{2}
$$

Cada presa fue medida con una regla Vernier $(0.1 \mathrm{~mm})$. Para todos los datos totales de la especie se estimó la importancia de los ítems alimentarios mediante el Índice de Importancia (IVI) (Powell et al. 1990):

IVI $=[$ Porcentaje del número + Porcentaje del volumen + Porcentaje de la frecuencia]/3

Adicionalmente, con fines comparativos se estimó el IVI independientemente para juveniles, hembras y machos.

Se calculó el tamaño de muestra adecuado mediante la metodología propuesta por Hurtubia (1973). Como primer paso, esta metodología estima la diversidad de los datos obtenidos mediante el índice de Brillouin (Magurran \& McGill 2012), y a partir de estos datos elabora una curva acumulada de diversidad para identificar el punto donde la curva se estabiliza, que corresponde al tamaño de muestra adecuado para evaluar la diversidad trófica de la especie estudiada. Dado que el orden de entrada de los datos puede afectar el resultado final, como parte de la metodología convencional, se realiza una aleatorización en el orden de entrada de los datos de cada estómago (Hurtubia 1973). Para reducir este potencial efecto, en el presente estudio adicionalmente se realizaron 25 repeticiones de este procedimiento, y se calculó una curva de acumulación "promedio" de diversidad para identificar el tamańo de muestra adecuado para evaluar la dieta de $M$. thoracicus icae.

También se evaluó la probable relación entre el tamaño corporal (SVL) y el total de número de tipos de presas, volumen total consumido y volumen vegetal consumido, mediante un Análisis de Regresión (Zar 1999). Los residuos de estas regresiones fueron comparados mediante la prueba de Kruskal-Wallis (Zar 1999), 
Tabla 1. Dieta de Microlophus thoracicus icae en el valle del río Ica. Se indican los valores de número y porcentaje (N\%), volumen y porcentaje (V\%) y frecuencia y porcentaje (\%F) de items consumidos. IVI: Índice de Importancia. Se destacan en negritas los mayores valores por columna de porcentajes.

\begin{tabular}{lccccccc}
\hline \multicolumn{1}{c}{ ITEM } & Número & N\% & Volumen & V\% & Frecuencia & F\% & IVI \\
\hline Aranea & 15 & 1.9 & 105.3 & 0.2 & 15 & 42.9 & 15.0 \\
Escorpionida & 1 & 0.1 & 251.3 & 0.6 & 1 & 2.9 & 1.2 \\
Solifuga & 3 & 0.4 & 45.1 & 0.1 & 2 & 5.7 & 2.1 \\
Isopoda & 1 & 0.1 & 1.7 & 0.0 & 1 & 2.9 & 1.0 \\
Coleoptera & 29 & 3.7 & 2437.9 & $\mathbf{5 . 5}$ & 16 & 45.7 & 18.3 \\
Diptera & 4 & 0.5 & 12.7 & 0.0 & 4 & 11.4 & 4.0 \\
Hymenoptera (alados) & 7 & 0.9 & 171.1 & 0.4 & 5 & 14.3 & 5.2 \\
Hymenoptera (Hormigas) & 350 & $\mathbf{4 4 . 1}$ & 258.5 & 0.6 & 20 & $\mathbf{5 7 . 1}$ & $\mathbf{3 3 . 9}$ \\
Lepidoptera & 3 & 0.4 & 815.4 & 1.8 & 3 & 8.6 & 3.6 \\
Larvas de insectos & 76 & $\mathbf{9 . 6}$ & 9950.9 & $\mathbf{2 2 . 5}$ & 21 & $\mathbf{6 0 . 0}$ & $\mathbf{3 0 . 7}$ \\
PANI & 23 & 2.9 & 2136.7 & 4.8 & 14 & 40.0 & 15.9 \\
Otros Artrópodos & 6 & 0.8 & 72.8 & 0.2 & 3 & 8.6 & 3.2 \\
Muda (piel) & 1 & 0.1 & 136.7 & 0.3 & 1 & 2.9 & 1.1 \\
Material Vegetal & 275 & $\mathbf{3 4 . 6}$ & 27741.0 & $\mathbf{6 2 . 9}$ & 30 & $\mathbf{8 5 . 7}$ & $\mathbf{6 1 . 1}$ \\
\hline
\end{tabular}

para determinar si existen diferencias entre juveniles, hembras y adultos en estas tres variables después de retirado el efecto del tamaño corporal. Adicionalmente, se comparó entre juveniles, hembras y machos el número de hormigas y larvas de insectos, el volumen total consumido, y el número de tipos de ítems consumidos. También se analizaron las probables diferencias en las proporciones de material vegetal y animal consumidas entre juveniles, hembras y machos. Para los análisis mencionados se emplearon pruebas no paramétricas debido a que los datos no presentaron distribuciones normales. Como primer paso se empleó la prueba de Kruskal-Wallis, y al obtener diferencias significativas se empleó posteriormente la prueba de U de MannWhitney (Zar 1999). Las pruebas estadísticas fueron realizadas en el programa Systat 11.0 y Past 3.0 (Hammer et al. 2001). El nivel de significancia fue definido en $\alpha=0.05$.

Se realizó un análisis de componentes principales (PCA) (Gauch 1994), para identificar semejanzas o diferencias entre las dietas de juveniles, hembras y machos. Para este análisis se empleó la matriz de datos de volúmenes consumidos transformados a proporciones.

Se calculó la amplitud del nicho trófico mediante el Índice de Simpson (Krebs, 1999):

$$
\mathrm{B}=1 / \sum \rho_{i}{ }^{2}
$$

Donde $p_{i}$ es la proporción del item alimentar $i$. Para este estimado utilizamos los datos de volumen de cada presa consumida para juveniles, hembras y machos.

\section{Resultados}

Se colectaron un total de 35 individuos de $M$. thoracicus icae, 27 adultos (16 machos, 11 hembras) y 8 juveniles. Mediante la metodología de Hurtubia (1973) se identificó que a partir de 16 estómagos analizados se obtendrían datos representativos de la dieta de $M$. thoracicus thoracicus, proporcionando un adecuado respaldo para los análisis realizados a continuación.

En términos numéricos, el principal item en la dieta de $M$. thoracicus icae lo constituyeron las hormigas (44.1\%), seguido del material vegetal (34.6\%) y larvas de insectos (9.6\%) (Tabla 1). El material vegetal fue exclusivamente partes (foliolos y flores) de los árboles Prosopis spp. En términos volumétricos, el principal ítem fue el material vegetal (62.9\%), seguido por las larvas de insectos $(22.5 \%)$ y coleópteros $(5.5 \%)$. Según la frecuencia, el principal ítem fue el material vegetal (85.7\%), seguido de larvas de insectos $(60.0 \%)$ y hormigas $(57.1 \%)$. Los ítems más importantes para la dieta de $M$. thoracicus icae según el IVI fueron el material vegetal (61.1\%), seguido de las hormigas (33.9\%) y larvas de insectos (30.7\%) (Tabla 1). Por otro lado, considerando los estimados de IVI para juveniles, hembras y machos el ítem más importante para estas tres categorías fue el material vegetal $(50.2 \%, 45.7 \%$ y $77.2 \%$ respectivamente), seguido de las larvas de insectos para hembras y machos (37.9\% y $24.3 \%$ respectivamente), y por himenópteros alados en juveniles

Tabla 2. Indice de Importancia (IVI) para juveniles, hembras y machos de Microlophus thoracicus icae en el valle del río Ica. Se destacan en negritas los mayores valores por columna.

\begin{tabular}{lccc}
\hline & Juveniles & Hembras & Machos \\
\hline Aranea & 22.7 & 12.7 & 13.0 \\
Escorpionida & 0.0 & 0.0 & 2.5 \\
Solifuga & 4.7 & 0.0 & 2.3 \\
Isopoda & 0.0 & 0.0 & 2.2 \\
Coleoptera & 13.6 & 18.6 & 19.7 \\
Diptera & 13.7 & 0.0 & 2.2 \\
Hymenoptera (alados) & $\mathbf{3 1 . 6}$ & $\mathbf{2 3 . 9}$ & 13.2 \\
Hymenoptera (Hormigas) & 27.5 & 9.4 & $\mathbf{2 0 . 9}$ \\
Lepidoptera & 0.0 & 7.4 & 2.4 \\
Larvas de insectos & $\mathbf{3 0 . 0}$ & $\mathbf{3 7 . 9}$ & $\mathbf{2 4 . 3}$ \\
PANI & 4.8 & 22.4 & 15.9 \\
Muda (piel) & 0.0 & 3.4 & 0.0 \\
Otros Artrópodos & 5.3 & 6.5 & 0.0 \\
Material Vegetal & $\mathbf{5 0 . 2}$ & $\mathbf{4 5 . 7}$ & $\mathbf{7 7 . 2}$ \\
\hline
\end{tabular}


Tabla 3. Parámetros de la dieta de Microlophus thoracicus icae para juveniles, hembras y machos. Se indican el número de categorías de item alimentarios consumidos, volumen total y amplitud de nicho trófico mediante el índice de Simpson $\left(B_{i j}\right)$.

\begin{tabular}{lccc}
\hline & Juveniles & Hembra & Macho \\
\hline Número de Categorías & 4.1 & 3.6 & 3.9 \\
Volumen Total & 154.6 & 1939.6 & 1347.8 \\
Amplitud Nicho (Bij) & 1.39 & 1.52 & 1.57 \\
\hline
\end{tabular}

\section{(31.6\%) (Tabla 2).}

No se registraron relaciones significativas entre el tamaño corporal de los individuos estudiados y el número total de presas consumidos $\left(\mathrm{R}^{2}=0.03 ; \mathrm{p}=0.31\right)$, ni el volumen total consumido $\left(R^{2}=0.05 ; \mathrm{p}=0.20\right)$ y volumen vegetal consumido $\left(\mathrm{R}^{2}=0.11 ; \mathrm{p}=0.06\right)$. De forma similar, después de retirado el efecto del tamańo del cuerpo (residuos de regresiones anteriores), no se registraron diferencias significativas en el número total de presas consumidas (Test KW $=1.49 ; \mathrm{p}=0.47$ ), volumen total consumido (Test KW $=2.02 ; \mathrm{p}=0.36$ ) o volumen vegetal consumido (Test $\mathrm{KW}=3.74 ; \mathrm{p}=0.15$ ) entre juveniles, hembras y machos.

Los juveniles, hembras y machos no presentaron diferencias significativas en el número de hormigas (Test $\mathrm{KW}=2.61 ; \mathrm{p}=$ 0.27 ) o larvas de insectos consumidos (Test $\mathrm{KW}=0.17 ; \mathrm{p}=$ 0.92). Sin embargo, se registraron diferencias significativas en el volumen del material vegetal consumido por juveniles, hembras y machos (Test KW $=14.53$; $\mathrm{p}<0.01$ ), específicamente el volumen de material vegetal fue mayor para machos y hembras comparado con juveniles (Test $\mathrm{U}=12.00$; $\mathrm{p}<0.01$; Test $\mathrm{U}=$ 87.00; $\mathrm{p}<0.01$ respectivamente). No se registraron diferencias significativas entre el consumo de material vegetal entre machos $y$ hembras (Test $U=104.00 ; p=0.43$ ). Por otro lado, tampoco se registraron diferencias significativas en la proporción de material vegetal consumido por juveniles, hembras y machos (Test $\mathrm{KW}=1.12 ; \mathrm{p}=0.57)$.

De manera general, el número de ítems alimentarios consumidos (tipos de presas) fue similar entre juveniles, hembras y machos (Test KW= 0.54; p = 0.76) (Tabla 3). El volumen total consumido fue menor en juveniles comparado con las hembras (Test $\mathrm{U}=87.00 ; \mathrm{p}<0.01$ ) y machos (Test $\mathrm{U}=12.00$; $\mathrm{p}<0.01$ ), sin embargo, no se registraron diferencias entre el volumen total consumido por hembra y machos (Test $U=104.00$; $p<0.43$ ) (Tabla 3). Considerando este resultado, donde los juveniles consumen volúmenes menores de alimento que los adultos, se determinó emplear los datos volumétricos proporcionales para los análisis de PCA, para que los resultados no estuvieran influenciados por el volumen total consumido. Los resultados del PCA muestran que los componentes 1 y 2 agrupan el $80.6 \%$ de la varianza de los datos. Se observa una alta relación positiva entre el componente 1 y el material vegetal, y en menor proporción una relación negativa de este mismo componente con larvas de insectos y coleópteros (Fig. 1). También se puede observar una considerable variación en la dieta de juveniles, hembras y machos (Fig. 1), que muestran la ausencia de agrupamientos evidentes entre estos tres grupos de individuos, evidenciando una semejanza entre sus dietas.

La amplitud del nicho trófico para $M$. thoracicus icae fue de $\mathrm{B}_{\mathrm{ij}}=6.97$. No se registraron diferencias significativas entre las amplitudes del nicho entre juveniles, hembras y machos (Test $\mathrm{KW}=0.02 ; \mathrm{p}=0.99)$ (Tabla 3 ).

\section{Discusión}

A diferencia de la información conocida sobre la dieta de la mayoría de saurios tropiduridos y gecónidos del Desierto Costero peruano, la lagartija de los gramadales Microlophus thoracicus icae presenta un importante consumo de material vegetal, siendo este el ítem el más importante en la dieta de juveniles y adultos. Pough $(1973,1983)$ sugiere que en general que las lagartijas con pesos menores a 100 gramos serían insectívoras, y saurios con pesos mayores serían omnívoras o herbívoras. Sin embargo, para Rocha (1989) la mayoría de lagartijas denominadas herbívoras son probablemente omnívoras. Nuestros datos muestran que $M$. thoracicus icae es una lagartija omnívora, pero con una importante cantidad de material vegetal en su dieta.

Según Dixon y Wrigth (1975) los adultos de M. thoracicus icae se alimentan casi exclusivamente de materia vegetal (hojas, flores y frutos inmaduros). Nuestros resultados muestran la im-

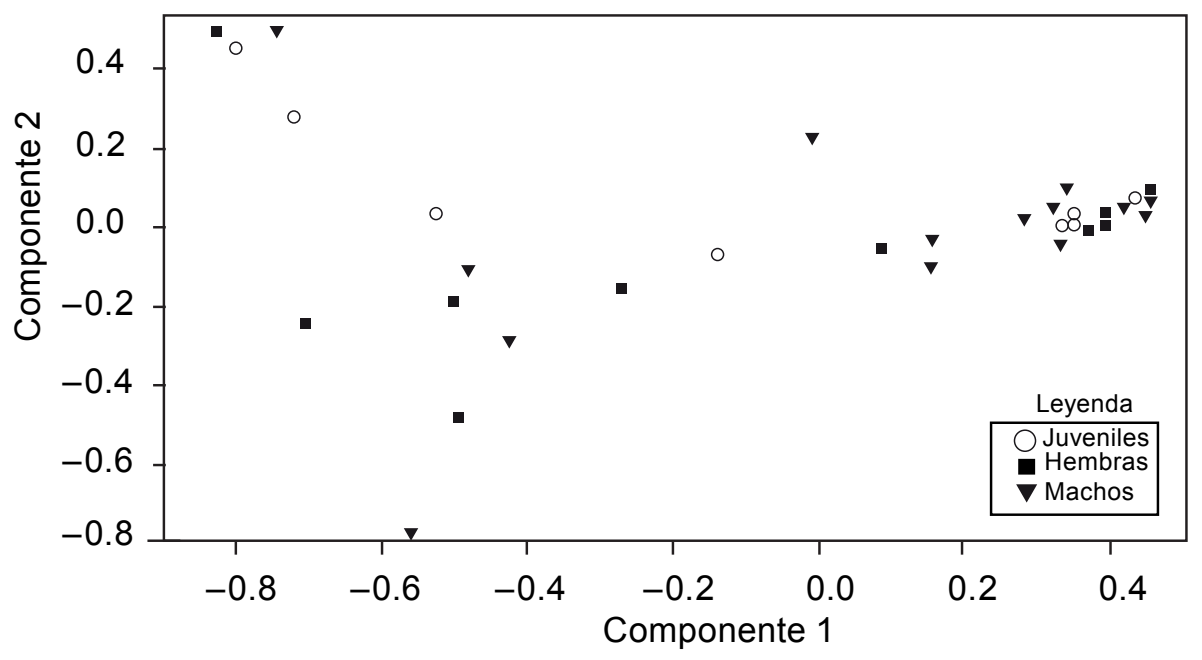

Figura 1. Análisis de Componentes Principales para la dieta de Microlophus thoracicus icae en el valle del río Ica. 
portancia de otros ítems como larvas de insectos e himenópteros (hormigas) en la dieta del adulto de este saurio, y además muestran que en juveniles también es importante el consumo de material vegetal.

Los resultados presentados evidencian la importancia del material vegetal para la dieta de $M$. thoracicus icae, sin embargo no se observa una marcada variación ontogenética en su dieta, como las reportadas en otras especies de saurios sudamericanos, como por ejemplo algunas especies de los géneros Tupinambis (Dessem 1985) y Dracaena (Estes \& Williams 1984). Las diferencias en $M$. thoracicus icae serían más sutiles, debido a las considerables semejanzas entre las dietas de juveniles y adultos, como por ejemplo en el consumo de hormigas, larvas de insectos, número de ítems, amplitud de nicho trófico, y la importancia del material vegetal, que en conjunto se reflejarían en la ausencia de agrupamientos marcados en el análisis multivariado. Estas semejanzas se deberían principalmente a la similitud en la oferta de recursos en estos individuos sintópicos, especialmente en ecosistemas desérticos, donde esta oferta es considerablemente restringida. Por otro lado, algunas de las diferencias registradas, como en el volumen total de presas consumidas, serían consecuencia directa de los diferentes tamaños corporales entre adultos y juveniles. Esta afirmación es reforzada por la ausencia de diferencias importantes entre las dietas de hembras y machos.

Considerando la importancia de los invertebrados en la dieta de adultos y del material vegetal en juveniles, podríamos afirmar que $M$. thoracicus icae es un omnívoro que presentaría gran plasticidad en su dieta, y que estaría influenciada directamente por la variación local de la oferta de los recursos alimenticios; es decir la amplia variación en su dieta, transitando desde insectívoro hacia herbívoro, seria consecuencia de las variaciones en la oferta del medio. Otro resultado que apoyaría esta hipótesis de gran variabilidad en la dieta de $M$. thoracicus icae, es la considerable amplitud registrada en el nicho trófico $\left(\mathrm{B}_{\mathrm{ij}}=6.97\right)$. La diversidad en la dieta reportadas para otras especies de saurios del desierto costero peruano son menores, como por ejemplo para Phyllodactylus angustidigitus $\left(\mathrm{B}_{\mathrm{ij}}=4.7\right)$ y P. gerrhopygus $\left(\mathrm{B}_{\mathrm{ij}}=1.9\right)$ (Pérez \& Balta 2011), M. peruvianus $\left(\mathrm{B}_{\mathrm{ij}}=2.97\right.$ ) (Quispitúpac \& Pérez 2009), M. tigris $\left(\mathrm{B}_{\mathrm{ij}}=4.33\right)$ (Pérez 2005), Ctenoblepharys adespersa $\left(\mathrm{B}_{\mathrm{ij}}=4.55\right)$ (Pérez \& Balta 2007).

En especies de saurios donde se reporta una variación ontogenética marcada en la dieta, se ha registrado un cambio del consumo de invertebrados en su etapa juvenil a un consumo principal o totalmente herbívoro en la etapa adulta (a partir de Mercolli \& Yanoski 1994; Kiefer \& Sazima 2002). También se observan importantes cambios asociados, como variación en el tipo de dientes y diferencias importantes en la comunidad de helmintofauna reportada para especies herbívoras y carnívoras (Petter \& Quentin 1976, Roca 1999). En M. thoracicus icae no se observan cambios en los tipos de dientes (J. Pérez obs. personal), hecho que reforzaría la hipótesis de una gran plasticidad en la dieta de esta especie, donde un cambio en la dentición restringiría su consumo de material vegetal o animal en alguna etapa de su vida. No obstante, es necesario determinar las potenciales consecuencias del consumo de una dieta tan variada por parte de $M$. thoracicus icae, como presentar características particulares en sus endoparásitos, estrategias y eficiencia de forrajeo, termorregulación, morfología, entre otros.

Como consecuencia de lo mencionado, podemos concluir que es necesario realizar evaluaciones adicionales para analizar como los cambios de la oferta de recursos a nivel de microhábitat y estacionales influenciarían el consumo de material vegetal y animal en $M$. thoracicus icae. Por otro lado, la estrecha relación reportada entre $M$. thoracicus icae y la vegetación, especialmente con los bosques de huarangos (Prosopis spp.), evidencia la alta sensibilidad de esta especie de saurio a impactos sobre este tipo de bosque. Por lo tanto, la sobreexplotación de los recursos naturales en el valle de Ica, que ha producido la disminución de ciertos recursos vegetales como el huarango (Roque \& Cano 1999), afectaría negativamente las poblaciones y el estado de conservación de $M$. thoracicus icae.

\section{Agradecimientos}

A Katya Balta por su ayuda en el análisis de los datos. A Edith Suazo, Angélico Asenjo, Luis Alza por la ayuda durante el trabajo de campo. A Hamilton Beltrán y José Roque por la ayuda en la identificación de plantas y bibliografía sobre la flora del área. A la Municipalidad de Ocucaje por el apoyo logístico durante la evaluación.

\section{Literatura citada}

Carrillo N. \& J. Icochea. 1995. Lista taxonómica preliminar de los reptiles vivientes del Perú. Publicaciones del Museo de Historia Natural de la Universidad Nacional Mayor de San Marcos Serie A: Zoología (49): 1-27.

Dessem D. 1985. Ontogenetic changes in the dentition and diet of Tupinambis (Lacerta:Teiidae). Copeia: 245 - 247. http:// dx.doi.org/10.2307/1444823

Estes R. \& E. Williams. 1984. Ontogenetic variation in the molariform teeth of lizards. Journal of Vertebrate Paleontology 4:96107. http://dx.doi.org/10.1080/02724634.1984.10011989

Dixon J. \& J. Wright. 1975. A review of the lizards of the iguanid genus Tropidurus in Peru. Contribution in Science. The Natural History Museum of los Angeles. 40 pp.

Dunham A.E. 1983. Realized niche overlap, resource abundance and intensity of interspecific competition. In R.B. Huey, E.R. Pianka and T.W. Schoener (eds.), Lizard Ecology. Harvard University Press, Cambridge, Massachusetts, U.S.A. pp 261280. http://dx.doi.org/10.4159/harvard.9780674183384. c15

Frost D.R. 1992. Phylogenetic analysis and taxonomy of the Tropidurus group of lizards (Iguania: Tropiduridae). American Museum Novitates 3033:1-68. http://hdl.handle.net/2246/4997

Gauch H.G. 1994. Multivariate analysis in community ecology. Cambridge University Press. USA. 298 pp.

Hammer Ø., D.A.T. Harper \& P.D. Ryan. 2001. Past: Paleontologica Statistics Software Package for education and data analysis. Paleontologia Electronica 4:1-9.

Huey R.B, E.R. Pianka \& T.W. Schoener. 1983. Lizard Ecology. Harvard University Press, Cambridge, Massachusetts, U.S.A. 501 pp. http://dx.doi.org/10.4159/harvard.9780674183384

Hurtubia J. 1973. Trophic diversity measurement in sympatric predatory species. Ecology 54(4): 885-890. http://dx.doi. org/10.2307/1935684

Icochea J. 1998. Lista roja preliminar de los anfibios y reptiles amenazados del departamento de Lima. En: Los Pantanos de Villa. Biología y Conservación. Editores: A. Cano y K. Young. Serie de Divulgación del Museo de Historia Natural de la Universidad Nacional Mayor de San Marcos. 217-229.

Kiefer M.C. \& I. Sazima. 2002. Diet of juvenile tegu lizard Tupinambis merianae (Teiidae) in southeastern Brazil. Amphibia-Reptilia 23:105-108.

Krebs C. 1999. Ecological Methodology. Addison Wesley Longman.

Magurran A.E. \& B.J. McGill. 2012. Biological Diversity: Frontiers in measurement and assessment. Oxford University Press, p. 345.

Mercolli C. \& A.A. Yanoski, 1994. Diet of Tupinambis teguixin in 
the eastern chaco of Argentina. Herpetologica 4: 15-19.

Pefaur J. \& E. López-Tejeda. 1983. Ecological notes on the lizard Tropidurus peruvianus in southern Peru. Journal of Arid Environments. 6:155-160.

Pérez Z. J. 2005. Ecologia de Duas Espécies de Lagartos Simpatricos em uma Formaçáo Vegetal de Lomas no Deserto Costeiro Peruano Central. Dissertaçáo de Mestrado. Universidade do Estado do Rio de Janeiro (UERJ). Rio de Janeiro. Brasil.

Pérez Z. J. \& K. Balta. 2006. Ecología de la comunidad de saurios diurnos de la Reserva Nacional de Paracas, Ica, Perú. Revista Peruana de Biología. 13(3): 169-176. http://dx.doi. org/10.15381/rpb.v13i3.2334

Pérez Z. J. \& K. Balta. 2011. Ecología de los gecónidos Phyllodactylus angustidigitus y P. gerrhopygus (Sauria: Phyllodactylidae) en la Reserva Nacional de Paracas, Ica, Perú. Revista Peruana de Biología 18(2): 217-223. http://dx.doi.org/10.15381/ rpb.v18i2.232

Pérez Z. J. \& J. Jahncke. 1998. Saurios como consumidores de ectoparásitos de aves guaneras. Boletín del Instituto del Mar de Perú. 81-86.

Pérez Z. J., L. Echevarría, S. Álvarez, A. Vera, G. Alarcón \& M. Andía. 2012. Ecología trófica de la lagartija Stenocercus modestus (Squamata: Tropiduridae) en una zona urbana, Lima, Perú. Revista Peruana de Biología. 19(3): 319 - 322. http://dx.doi. org/10.15381/rpb.v19i3.1047

Petter A.J. \& J. C. Quentin. 1976. Keys to genera of the Oxyuroidea. Pp. 1-30, En: Anderson, R.C., Chabaud, A.G. y Willmott, S. (eds.), CIH Keys to the Nematode Parasites of Vertebrates. CAB. International, London.

Pisani G.R. \& J. Villa. 1974. Guía de Técnicas de preservación de anfibios y reptiles. Lawrence. Society for the Study of the
Amphibians and Reptiles. 24pp.

Pollack L., W. Zelada, A. Tirado \& L. Pollack. 2007. Hábitos alimentarios de Dicrodon guttulatum, "ca-án" (Squamata: Teiidae) en Garrapón, Paiján. Arnaldoa 14(2):283- 291.

Pough F.H. 1973. Lizard energetics and diet. Ecology. 54:837-844. http://dx.doi.org/10.2307/1935678

Pough F.H. 1983. Ambhibians and reptiles as low energy systems. Behavioral Energetics, pp 141-188.

Powell R., J.S. Parmelee, M.A. Rice \& D.D. Smith. 1990. Ecological observations on Hemidactylus brooki haitianus Meerwarth (Sauria : Gekkonidae) from Hispaniola. Caribean Journal of Science 26: 67-70.

Roca V. 1999. Relación entre las faunas endoparásitas de reptiles y su tipo de alimentación. Revista Española de Herpetología 13: 101-121.

Rocha C.F.D. 1989. Diet of a Tropical Lizard (Liolaemus lutzae) of Southeastern Brazil. Journal of Herpetology. 23 (3):292-294. http://dx.doi.org/10.2307/1564451

Roque J. \& A. Cano. 1999. Flora vascular y vegetación del valle de Ica, Perú. Revista Peruana de Biología. 6 (2): 185-195. http:// dx.doi.org/10.15381/rpb.v6i2.8314

Quispitúpac E. \& J. Pérez. 2009. Dieta de la lagartija de las playas Microlophus peruvianus en la playa Santo Domingo. Reserva Nacional de Paracas, Ica. Revista Peruana de Biología 15(2): 129-130. http://dx.doi.org/10.15381/rpb.v15i2.1739

Van Leeuwen J.P., A. Catenazzi \& M. Holmgren. 2011. Spatial, Ontogenetic, and Sexual Effects on the Diet of a Teiid Lizard in Arid South America. Journal of Herpetology, 45(4):472-477. http://dx.doi.org/10.1670/10-154.1

Zar J. H. 1999. Biostatistical analysis. 4 ed. Upper Saddle River. Prentice Hall. 